\title{
Gas Kinematics in the Multiphase Circumgalactic Medium
}

\author{
Nikole M. Nielsen ${ }^{1}$, Glenn G. Kacprzak ${ }^{1}$, Christopher W. Churchill ${ }^{2}$, \\ Michael T. Murphy ${ }^{1}$, Sowgat Muzahid ${ }^{3}$, Jane C. Charlton ${ }^{3}$ and \\ Jessica L. Evans ${ }^{2}$ \\ ${ }^{1}$ Centre for Astrophysics and Supercomputing, \\ Swinburne University of Technology, Hawthorn, Victoria 3122, Australia \\ email: nikolenielsen@swin.edu.au \\ ${ }^{2}$ Department of Astronomy, New Mexico State University, \\ Las Cruces, NM 88003, USA \\ ${ }^{3}$ Department of Astronomy \& Astrophysics, The Pennsylvania State University, \\ State College, PA 16801, USA
}

\begin{abstract}
We use high-resolution Keck, VLT, or Hubble Space Telescope spectra of background quasars to examine the kinematic properties of the multiphase, metal-enriched circumgalactic medium in the outskirts of galaxies at $0.08<z_{\text {gal }}<1.0$, focusing on the low-ionization Mg II and high-ionization Ovi doublets. The absorption kinematics of low-ionization gas in the circumgalactic medium depend strongly on the star formation activity and the location about the host galaxy, where the largest velocity dispersions are associated with blue, face-on galaxies probed along the minor axis. Conversely, high-ionization gas kinematics are independent of galaxy star formation activity and orientation.
\end{abstract}

Keywords. galaxies: halos, galaxies: evolution, quasars: absorption lines

\section{Introduction}

The bound gaseous halo surrounding galaxies, i.e., the circumgalactic medium (CGM), is a massive $\left(\sim 10^{9} M_{\odot}\right.$; Tumlinson et al. 2011) reservoir of diffuse multiphase gas, some of which will become the fuel for future star formation. It is a crucial component in the baryon cycle, where gas accretes onto, outflows from, and recycles back onto galaxies through the CGM. Studying the CGM is most easily done by examining absorption in background quasar spectra. The MgII $\lambda \lambda 2796,2803$ doublet is commonly studied and has been associated with accretion/recycling (e.g., Rubin et al. 2012), outflows (e.g., Bouché et al. 2012), and merging satellite galaxies (e.g., Martin et al. 2012). This ion, and the higher ionization OVI $\lambda \lambda 1031,1037$ doublet, have been found to be preferentially located along the projected major and minor axes of galaxies (e.g., Bordoloi et al. 2011; Bouché et al. 2012; Kacprzak et al. 2012, 2015; Lan et al. 2014), hinting at inflowing and outflowing material, respectively.

Additional work found that the equivalent width (EW) of the MgII ion depends strongly on the inclination and/or the azimuthal angle of the host galaxy, and associated larger EWs with outflows (e.g., Kacprzak et al. 2011; Bordoloi et al. 2011; Bouché et al. 2012). The EW is a combination of the velocity spread of absorption and the column density, so outflows may result in large gas velocities, large amounts of material, or both. To further understand the EW differences and provide insight into the motions of gas involved in the baryon cycle, we examine the kinematics of the absorbers as a function of galaxy color (star formation rate, SFR, proxy), inclination, and azimuthal angle. The majority of the work presented here is summarized from Nielsen et al. (2015). 


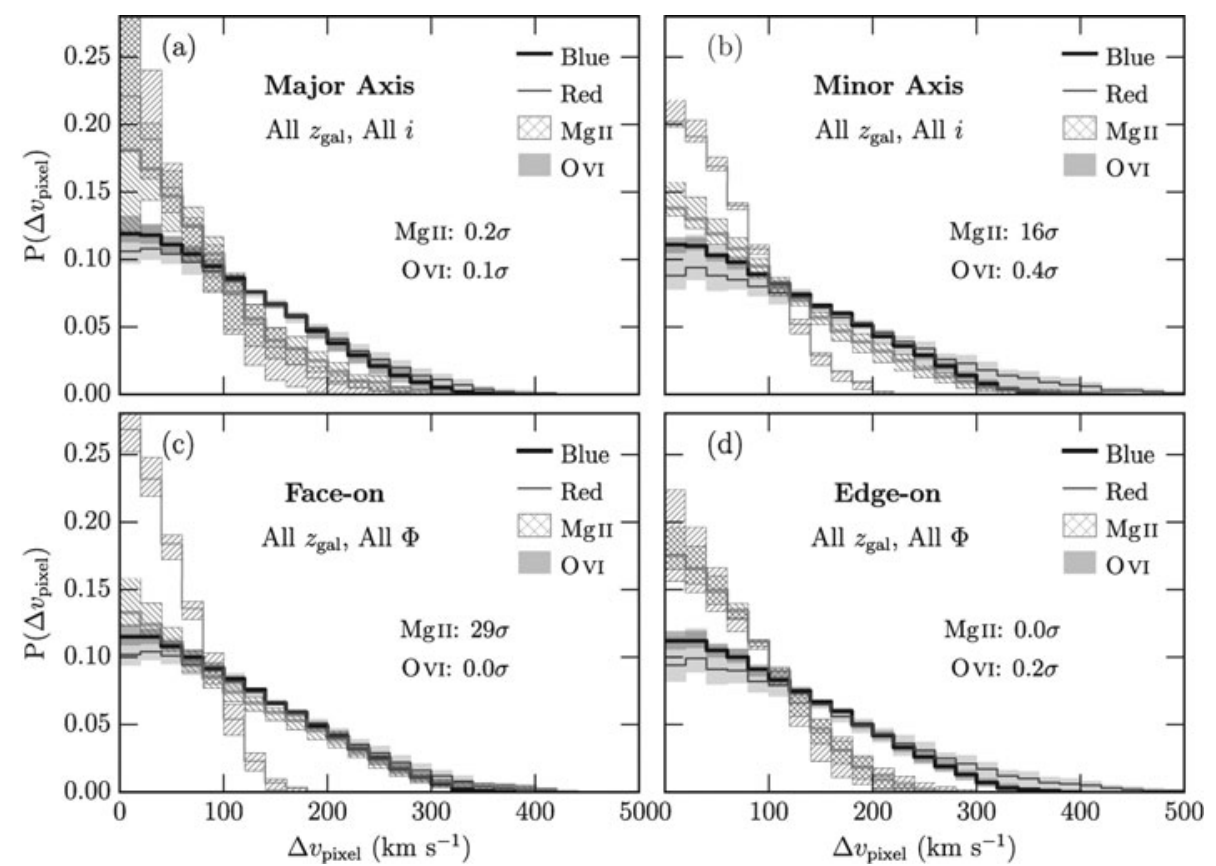

Figure 1. Blue and red galaxy TPCFs for O vi absorbers (solid shading) and for Mg II absorbers (hatched shading). Thicker lines correspond to blue galaxies while thinner lines correspond to red galaxies. Panels (a) and (b) compare galaxies probed along the projected major and minor axes, respectively. Panels (c) and (d) are for galaxies in face-on or edge-on orientations, respectively. The significance of a chi-squared test comparing blue and red galaxies for each ion and subsample are listed in each panel.

\section{Absorber Kinematics}

The Sample: We use two separate data sets: one for absorber-galaxy pairs in which Mg II was detected, and one where O vi was detected, with some overlap between the sets. For MgII, we have 30 pairs whose absorption is detected in high-resolution HIRES/Keck or UVES/VLT quasar spectra (see Nielsen et al. 2015, 2016). With OvI, we have 30 pairs from our "Multiphase Galaxy Halos" large Hubble Space Telescope (HST) program whose absorption is detected in COS/HST quasar spectra (Kacprzak et al. 2015).

All galaxies range in redshift from $0.08<z_{\text {gal }}<1.0$ and are probed within $D<200 \mathrm{kpc}$ by a background quasar. Each galaxy was imaged with WFPC2/HST and their morphologies, quasar-galaxy azimuthal angles, and inclinations were modeled using GIM2D (full details of galaxy modeling are described in Kacprzak et al. 2011, 2015). Each galaxy also has a $B-K$ color (a SFR proxy). With these data, we make the following subsamples: "blue" or "red" galaxies (median of $B-K=1.4$ for MgII or $B-K=1.66$ for O VI), "major axis" or "minor axis" $\left(\Phi<45^{\circ}\right.$ and $\Phi \geqslant 45^{\circ}$, respectively), and "face-on" or "edge-on" (cut by $i=57^{\circ}$ for $\mathrm{Mg}$ II or $i=51^{\circ}$ for O vi).

Pixel-velocity TPCF: The pixel-velocity two-point correlation function (TPCF) describes the velocity dispersion of absorption. Full details are described in Nielsen et al. $(2015,2016)$. A velocity of $v=0 \mathrm{~km} \mathrm{~s}^{-1}$ in the spectrum indicates the optical depth weighted median of absorption (as opposed to the galaxy redshift). Uncertainties on the TPCFs are calculated using a bootstrap analysis and we quantify the differences between TPCFs for subsample pairs with a chi-squared test that takes into account the uncertainties. The TPCFs and their chi-squared test results are presented in Figure 1. 
Low-ionization Absorbers: As shown in Figure 1 (TPCFs with hatched shaded uncertainties), Mg II absorbers depend strongly on the galaxy orientation and color. Galaxies that are bluer (more star-forming) have large absorber velocity dispersions when probed along the minor axis (panel (b)) and for those in face-on orientations (panel (c)). Bipolar outflows driven by stellar feedback are commonly detected for these orientations (e.g., Rubin et al. 2014), thus the large velocity dispersions in the face-on subsample can be explained as the outflows being pointed towards the observer, where the velocity of the gas is maximized. Redder galaxies (less star forming) have very narrow velocity dispersions when in face-on orientations, possibly due to the lack of star formation driven outflows.

The velocity structure of Mg II absorbers does not depend on star formation rate when probed along the projected major axis (panel (a)) nor for edge-on orientations (panel (d)), and the velocity dispersions are smaller than in panels (b) and (c). These orientations are where accreting and rotating gas are more likely to be found, because they are where gas velocities for these processes are maximized (e.g., Danovich et al. 2015).

High-ionization Absorbers: Conversely, for the TPCFs with solid shaded uncertainties in Figure 1, OvI absorber kinematics do not depend on galaxy orientation or color (star formation activity). The OvI absorbers have larger velocity dispersions than MgII for nearly all subsample comparisons. Only two MgII subsamples are comparable to Ovi: the blue, minor axis (panel (b)) and blue, face-on (panel (c)) subsamples.

Since the high-ionization gas kinematics do not depend on galaxy properties, and have similar velocity dispersions as low-ionization absorbers in orientations where outflows are likely, this may hint at Ovi being associated with previous outflow activity. We note that O VI is only observed around star forming galaxies (Tumlinson et al. 2011), unlike MgII. Thus OVI absorbers appear to trace a different component of the CGM than MgII.

\section{Summary}

Low-ionization gas in the CGM is sensitive to the host galaxy star formation and outflow activity, whereas the high-ionization gas is not. This may indicate that the two ions trace different components of the CGM. A next step in understanding the role of the CGM in the baryon cycle would be to obtain metallicities of the absorbers (the equivalent width also depends on gas metallicities) to pinpoint whether the gas is more likely metal-enriched outflows or more metal-poor accretion/recycling.

\section{Acknowledgments}

NMN would like to thank the IAU for a travel grant.

\section{References}

Bordoloi, R., Lilly, S. J., Knobel, C., et al. 2011, ApJ, 743, 10

Bouché, N., Hohensee, W., Vargas, R., et al. 2012, MNRAS, 426, 801

Danovich, M., Dekel, A., Hahn, O., et al. 2015, MNRAS, 449, 2087

Kacprzak, G. G., Churchill, C. W., Evans, J. L., et al. 2011, MNRAS, 416, 3118

Kacprzak, G. G., Churchill, C. W., \& Nielsen, N. M. 2012, ApJL, 760, L7

Kacprzak, G. G., Muzahid, S., Churchill, C. W., et al. 2015, ApJ, 815, 22

Lan, T. W., Ménard, B., \& Zhu, G. 2014, ApJ, 795, 31

Martin, C. L., Shapley, A. E., Coil, A. L., et al. 2012, ApJ, 760, 127

Nielsen, N. M., Churchill, C. W., Kacprzak, G. G., et al. 2015, ApJ, 812, 83

Nielsen, N. M., Churchill, C. W., Kacprzak, G. G., et al. 2016, ApJ, 818, 171

Rubin, K. H. R., Prochaska, J. X., Koo, D. C., et al. 2012, ApJ, 747, L26

Rubin, K. H. R., Prochaska, J. X., Koo, D. C., et al. 2014, ApJ, 794, 156

Tumlinson, J., Thom, C., Werk, J. K., et al. 2011, Science, 334, 948 